\author{
M.B. Pasticci \\ G. Mancini \\ L.M. Lapalorcia \\ S. Morosi \\ N. Palladino \\ M. Zucchini \\ F. Baldelli
}

\section{Prosthetic infections following total knee arthroplasty: a six-year prospective study (1997-2002)}

Received: 28 September 2006

Accepted: 21 January 2007

Published online: 5 March 2007

M.B. Pasticci $(\varangle) \cdot$ L.M. Lapalorcia S. Morosi • N. Palladino • F. Baldelli Infectious Diseases Section Department of Experimental Medicine and Biochemical Sciences

University of Perugia

Perugia, Italy

E-mail: pasticci@unipg.it

G. Mancini $•$ M. Zucchini

Orthopaedics and Traumatology Section

Department of Surgical Sciences

University of Perugia

Perugia, Italy

\begin{abstract}
This paper reports on a prospective observational study that evaluated the frequency of prosthetic infections after total knee arthroplasty. During a six-year observational period, 171 patients underwent knee arthroplasty. Single shot prophylaxis with teicoplanin was administered to all patients. Nine patients $(5.3 \%)$ had a followup of less than four weeks; the remaining 162 had a follow-up of at least 12 weeks. Of these, 155 completed the 24-month observational period. In the end, three patients developed early prosthetic joint infection which produced infection rates of $1.85 \%$ and $1.93 \%$ when the follow-up was $\geq 12$ weeks
\end{abstract}

and $\geq 24$ months, respectively. The mean time from surgery to infection was 54 days (range, 14-87). All three infections were caused by Staphylococcus aureus susceptible to methicillin. Fever, pain, effusion and secretion were present in all cases. In this cohort of patients, no cases of delayed infection were observed. Thorough reporting is the first step in reducing the incidence of post-surgical infective complications and can contribute to more productive prophylactic protocols.

Key words Prospective study • Prosthetic knee surgery • Prosthetic knee infection

\section{Introduction}

Prosthetic joint infections (PJIs) are infrequent but serious complications after total knee arthroplasty. PJIs are reported in approximately $1.5 \%-2.5 \%$ of knee arthroplasty cases $[1,2]$. Recently, some authors have observed rates as low as $0.5 \%$ after applying aggressive control measures, such as use of laminar air flow rooms, body exhaust suits, and correct peri-operative antibiotic prophylaxis and care [3]. PJIs remain one of the most serious complications associated with a substantial increase in morbidity leading to unnecessarily prolonged hospital stays and increased monetary costs [4]. Moreover, PJIs can be aggravated by a mortality rate as high as $2.5 \%$ [5].

The aims of this study were to examine the frequency of infections after knee arthroplasty in our institution and to characterize clinical and microbiologic aspects of the prosthetic joint infections observed.

\section{Materials and methods}

This was a prospective observational study, conducted at the Department of Orthopaedics and Traumatology of the University of Perugia. All patients without a previous history of PJI who underwent knee replacement from January 1997 to December 2002 were included.

Demographic characteristics, underlying illnesses, risk factors, and antibiotic prophylaxis were recorded. After surgery, each patient was examined whenever required or, in the absence of symptoms, every three months over the first post-surgical year. Thereafter, follow-up was obtained and recorded by ambulatory visit, letter or telephone the following year. 
All surgical procedures were performed in a standard operation theater with standard air conditions (not ultra-clean air) and standard clothing. The implants were fixed using cement without antibiotics and the patella was never resurfaced. In all the cases, tourniquets were inflated at the beginning of the procedure and were released after cementing the components. Tourniquets were inflated for mean times of 65 minutes (range, 50-90) and 50 minutes (range, 40-65) for total knee and mono-compartmental arthroplasties, respectively. The mean operating times from skin incision to wound suture were 85 minutes (range, 70-120) for total knee arthroplasty and 65 minutes (range, 55-80) for monocompartmental arthroplasty. Peri-operative prophylaxis consisted of intravenous teicoplanin (400 mg) one hour prior to surgery, as recommended $[9,11]$. Antibiotic-impregnated bone cement was never used.

In order to define cases of PJIs, standard clinical, radiological and microbiologic criteria were used [4, 6]. PJI was classified as acute when it developed within three months after surgery, or delayed when occurring within 24 months of arthroplasty $[7,8]$.

Risk factors for PJI were distinguished on the basis of whether they were patient-dependent or operation-related. The former included: rheumatoid arthritis, diabetes mellitus, malignancy, steroid use, chronic renal insufficiency, pyuria $(>10$ WBCs per high power field), bacteruria $\left(\geq 10^{5}\right.$ colonies of bacte$\mathrm{ria} / \mathrm{ml}$ urine), obesity (weight $>20 \%$ ideal body weight), malnutrition (albumin level $<3.0 \mathrm{~g} / \mathrm{dl}$ ) and prior knee surgery. The latter were: surgical site infection, surgical site hematoma, wound dehiscence and nosocomial infection of other sites [4, 8, 12].

\section{Results}

During the six-year study period (1997-2002), 171 patients underwent total knee arthroplasty (Table 1). The patients had a mean age of 71 years (range, 37-82 years) and $109(63 \%)$ were female. Nine patients $(5.3 \%)$ had a follow-up of less than four weeks; the remaining $162 \mathrm{had}$ a follow-up $\geq 12$ weeks, and 155 of these were followed for at least 24 months. Degenerative joint disease was the cause of joint replacement for all 171 patients. Specifically, 169 had arthrosis and two had septic arthritic outcomes. The most frequently reported underlying diseases were hypertension (81 patients) and cardiopathy (10 patients). The most common host risk factors for infection were diabetes and obesity.

Overall, 3 PJIs were observed. Considering the 155 patients with the two-year follow-up, the infection rate was $1.93 \%$. The mean time from surgery to infection was 54 days (range, 14-87 days). All three patients with infections were women who had had a bi-compartmental knee prosthesis implanted. None of these patients had reported having knee infection. Their mean age was 78 years (range, 71-80). One patient had hypertension. Obesity was present in two and one had insulin-dependent diabetes.
Table 1 Clinical characteristics of 171 patients who underwent total knee arthroplasty for degenerative joint disease

$\begin{array}{lr}\text { Male, } n(\%) & 62(37) \\ \text { Age, years } & 71(37-82) \\ \text { Underlying diseases, } n(\%) & \\ \quad \text { Hypertension } & 81(47) \\ \quad \text { Cardiopathy } & 10(6) \\ \text { Risk dactors for PJI, } n(\%) & \\ \quad \text { Patient-related } & \\ \quad \text { Diabetes } & \\ \quad \text { Obesity } & 24(14) \\ \quad \text { Operation-releated } & 12(7) \\ \quad \text { Surgical wound infection } & \\ \quad \text { Urinary tract infection } & 2(1) \\ \text { Prosthesis } & 1(1) \\ \quad \text { Monocompartmental } & \\ \quad \text { Bi-compartmental } & 9(5) \\ \text { Follow-up } & 162(95) \\ \geq 3 \text { months } & \\ \geq 24 \text { months } & 162(95) \\ \end{array}$

a Values are mean (range)

$P J I$, prosthetic joint infection

Methicillin-susceptible Staphylococcus aureus (MSSA), was the only microorganism identified in all three cases.

A few days after surgery, two of the patients manifested fever, effusion, wound dehiscence and drainage. Wound secretions grew methicillin-susceptible $S$. aureus in both cases. Both strains showed the same antimicrobial susceptibility pattern of $S$. aureus strains subsequently isolated from joint fluid and intra-operative bone biopsies. The third patient had an E. coli nosocomial urinary tract infection in the postoperative period. Nevertheless, this latter patient also developed $S$. aureus PJI characterized by fever, joint pain, effusion, erythema, and cutaneous draining sinus. For all three cases, elevated leukocyte count with an increased percentage of neutrophils, elevated erythrocyte sedimentation rate (ESR) and increased Creactive protein (CRP) were recorded.

All three patients were successfully treated with a combination of antimicrobial therapy and surgical treatments. In an attempt to avoid prosthesis removal, one of the first two patients with an infection that developed 14 days after undergoing knee arthroplasty initially was treated with arthrotomy, synovectomy and debridement of soft tissue. However, the infection was still present one year later, and therefore, the patient had to undergo a two-stage exchange arthroplasty. Currently, four years after the second arthroplasty, there are no signs of infection. Regarding the other two patients, surgery consisted of a two-stage exchange arthroprothesis and the patients have remained symptom-free for four and six years, respectively. 


\section{Discussion}

This prospective observational study evaluated the frequency of infections after total knee arthroplasty. Among the 171 patients who underwent knee arthroplasty over the six-year observational period, $162(94.7 \%)$ had a followup $\geq 12$ weeks and 155 completed the 24-month observational period. In the end, three patients had developed early prosthetic joint infection, giving infection rates of $1.85 \%$ and $1.93 \%$ for the $\geq 12$-week and $\geq 24$-month follow-ups, respectively. Both of these values are within the ranges reported in the literature $[1,2]$. However, it is difficult to compare different studies, since variances in patient characteristics, prophylactic regimens, and criteria for diagnosis and surveillance all influence infection rates. For this cohort of patients, follow-up was inferior or equal to 24 months while some peri-operative infections may have latency periods longer than 2 years. Thus, in this study the rate of delayed infections may have been underestimated.

$S$. aureus was the only etiologic agent isolated. $S$. aureus wound infection could have preceded prosthesis infection in two patients, since a few days after surgery they presented clinical signs of acute infection together with surgical wound dehiscence and drainage. Surgical site infection is a significant risk factor for prosthesis infection [4]. In the early postoperative period, the implanted biomaterial is more susceptible to bacterial infection and deep peri-prosthesis tissues are not suffi- ciently protected by host defense mechanisms [13, 14]. $S$. aureus is the most frequent microorganism causing deep early postoperative infections [8]. S. aureus possesses surface factors and virulence factors $[15,16]$ which usually take part in host colonization and disease $[1,6,8]$. Indeed, $S$. aureus PJIs are associated with more symptomatic presentation. All the three patients in this study manifested clinical signs of acute infections with above-normal ESR, CRP and leukocyte counts.

The presence of advanced age or insulin-dependent diabetes may have predisposed the patients to colonization with $S$. aureus $[15,16]$. Colonized patients undergoing surgery are considered to be at a higher risk for developing local and systemic staphylococcal complications [15-17].

All the $S$. aureus strains in these patients were multisusceptible methicillin-sensitive and resistant only to penicillin. This favorable antimicrobial susceptibility pattern leads us to hypothesize that the prosthesis contamination could have been autotransmitted in the peri-operative period rather than hospital-acquired.

During this study, the greatest challenges were encountered when trying to successfully trace patient data after surgery. Consequently, our results have induced us to (1) review the current operative procedures, (2) introduce a regional prosthesis registry with the active involvement of other health care personnel, and (3) prolong the observation period with the final aim of developing a better PJI detection system $[18,19]$.

\section{References}

1. Lentino JR (2003) Prosthetic joint infection: bane of orthopedicsts, challange for infectious disease specialists. Clin Infect Dis 36:1157-1161

2. Windsor RE (1991) Management of total knee arthroplasty infection. Orthop Clinic North Am 22:5331-5338

3. Peersman G, Laskin R, Davis J, Peterson M (2001) Infection in total knee replacement: a retrospective review of 6489 total knee replacements. Clin Orthop Relat Res 392:15-23

4. Berbari EF, Hanssen AD, Duffy MC et al (1008) Risk factors for prosthetic joint infection: case-control study. Clin Infect Dis 27:1247-1254

5. Fisman DN, Reilly DT, Karchmer AW, Goldie SJ (2001) Clinical effectiveness and cost-effectiveness of 2 management strategies for infected total hip atrthroplasty in the elderly. Clin Infect Dis $32: 419-430$
6. Zimmerli W, Trampuz A, Ochsner PE (2004) Prosthetic-joint infections. N Engl J Med 351:1645-1654

7. Nafziger DA, Saravolatz LD (1997) Infection in implantable prosthetic devices. In Wanzel RP (ed) Prevention and control of nosocomial infections. William \& Wilkins, Baltimore, pp 889-916

8. Tsukayama DT, Goldberg VM, Kyle R (2003) Diagnosis and management of infection after total knee arthoroplasty. J Bone Joint Surg Am 85[Suppl 1]:S75-S80

9. Gillespie WJ (1997) Prevention and management of infection after total joint replacement. Clin Infect Dis 25:1310-1317
10. Periti P, Stringa P, Mini E (1999) Comparative multicenter trial of teicoplanin versus cefazolin for antimicrobial prophylaxis in prosthetic joint implant surgery. Italian Study Group for Antimicrobial Prophylaxis in Orthopedic Surgery. Eur J Clin Microbiol Infect Dis 18:113-119

11. De Lalla F, Viola R, Pellizzer G et al (2000) Regional prophylaxis with teicoplanin in monolateral or bilateral total knee replacement: an open study. Antimicrob Agents Chemother 44:316-319

12. Atkins BL, Athanasou N, Deeks JJ et al (1998) Prospective evaluation of criteria for microbiological diagnosis of prosthetic-joint infection at revision arthroplasty. J Clin Microbiol 36:2932-2939 
13. Foster T, Hook M (2000) Molecular basis of adherence of Staphylococcus aureus to bio-materials. In: Waldgevel FA, Bisno AL (eds) Infection associated with indwelling medical devices, 3rd edn. American Society for Microbiology, Washington D.C., pp 27-39

14. Zimmerli W, Lew PD, Waldvogel FA (1984) Pathogenesis of foreign body infection: evidence for a local granulocyte defect. J Clin Invest 73:1191-1200
15. Waldvogel FA (2000) Gram positive cocci. Staphylococcus aureus including staphylococcal toxic shock. In: Mandell GL, Bennet JE, Dolin R (eds) Mandell, Douglas, and Bennett's Principles and practice of infectious diseases, 5th edn. Churcill Livingstone, Philadelphia, pp 2069-2092

16. Moreillon P, Que YA, Glauser M P (2005) Staphylococcus aureus including staphylococcal toxic shock. In: Mandell GL, Bennet JE, Dolin R (eds) Mandell, Douglas, and Bennett's Principles and practice of infectious diseases, 6th edn. Churchill Livingstone, Philadelphia, pp 2321-2351
17. Kalmeijer MD, van Nieuwland-Bollen E, Bogaers-Hofman D, de Baere GA (2000) Nasal carriage of Staphylococcus aureus is a major risk factor for surgical-site infections in orthopedic surgery. Infect Control Hosp Epidemiol 21:319-323

18. Condon RE, Schulte WJ, Malangoni MA, Anderson-Teschendorf MJ (1983) Effectiveness of a surgical wound surveillance program. Arch Surg 118:303-307

19. Schneeberg PM, Smits MH, Zick REF, Wille JC (2002) Surveillance as a starting point to reduce surgical-site infection rates in elective orthopaedic surgery. J Hosp Infect 51:179-184 